\title{
LOS DESAFÍOS DE LOS POSGRADOS EN CIENCIAS SOCIALES EN CHILE
}

\section{Introducción}

El problema central derivado de la constitución evolutiva de una sociedad mundial a inicios del siglo XXI, que pone en relación conflictiva contextos globales supranacionales con espacios regionales y territoriales de carácter estatal nacional, puede ser claramente observado en la evolución del sistema educativo y la especialización universitaria, fundamentalmente en la formación de posgrado. En este campo pueden encontrarse, paradójicamente, las mayores expectativas para la formación de capital humano adecuado a las semánticas desarrollistas del Estado nacional o de espacios regionales, y, a la vez, los mejores indicios de la desnacionalización del sistema educativo y su asociación con la formación de disciplinas que operan supranacionalmente sin una necesaria referencia territorial o regional.

El objetivo de nuestra investigación fue dar cuenta de las distintas dimensiones de este problema desde el punto de vista de la formación de posgrado en el campo de las ciencias sociales en Chile. A este ejercicio subyace la tesis siguiente: los distintos niveles de la sociedad mundial se constituyen en puntos de referencia para distintas orientaciones en la formación de posgrado en el área de las ciencias sociales en Chile. Los niveles territorial y regional orientan la formación de posgrado hacia campos de carácter temático en los que, más bien, se leen semánticas de carácter político-desarrollistas; el nivel supranacional de la sociedad mundial, en tanto, está incipientemente presente en el desarrollo de posgrados de carácter disciplinar con un interés manifiesto en la investigación científica y en los métodos y teorías de esos cuerpos de conocimiento. De este modo, la caracterización de los posgrados en Chile en esta área de las ciencias 
-aunque no sólo ahí- puede entenderse a través de la permanente operación de la distinción temático-profesional/disciplinar y los diversos énfasis con que ella se exprese en los programas de estudio y formación concretos.

Para dar cuenta de esta tesis se despliega, en primer lugar, la idea de sociedad mundial como interrelación ascendente y descendente entre el nivel global de la diferenciación funcional, los espacios regionales y los territoriales (II), para luego, sobre este marco, desarrollar la idea de complejidad y emergencia como horizonte de la formación de posgrado (III), y dar cuenta de las conclusiones centrales que emergen del vínculo entre formación de posgrado en ciencias sociales y sociedad mundial, observada desde la diferencia directriz temático-profesional/disciplinar (IV). Sigue el texto con la especificación de propuestas regulatorias para el desarrollo de la formación de posgrados en ciencias sociales en Chile (V) y concluye con una breve síntesis de la argumentación general (VI).

\section{Contexto global, región y territorio en la sociedad mundial}

La sociedad mundial no se entiende únicamente en el sentido formal de "unidad de todas las comunicaciones posibles" (Luhmann, 1997a, 2005), tampoco de modo exclusivo como "diferenciación funcional y expansión organizacional que integra lo territorial y lo regional en la diferenciación global" (Stichweh, 2000) ni sólo como "una sociedad mundial de actores laterales universales y sistemas basada en sociedades nacionales territoriales" (Willke, 2005). Todos estos acercamientos aportan sin duda a la definición aquí propuesta: articulación emergente de los niveles territorial, regional y global, es decir, la articulación de: a) territorios nacionales y espacios locales (territorio) con b) organizaciones, instituciones y actores supranacionales (región) y c) espacios operacionales de la diferenciación funcional y los sistemas simbólicos (global). 
Diferenciación funcional es el modo característico de organización de la sociedad moderna, es decir, sistemas como la educación, el derecho, la política, con lógicas de funcionamiento que traspasan las fronteras nacionales y que posibilitan respuestas institucionales localmente diferenciadas pero comparables y operativamente homogéneas entre sí. Son fundamentalmente los sistemas laterales mundiales (Willke, 2001) -es decir, las organizaciones, los actores transnacionales y las redes de coordinación- los que permiten a la vez homogeneidad y especificidad en la sociedad mundial (Stichweh, 2000). La sociedad moderna puede ser crecientemente descrita como sociedad mundial. Ella supone una comprensión desterritorializada de lo social como comunicación que integra todos los posibles horizontes de mundo -sea de individuos o colectivos- como extensiones de un único sistema comunicativo universal en el que se incluyen todas las posibilidades de comunicación (Luhmann, 1971, 1997a, 2005). En otras palabras, no hay comunicación más allá de la sociedad mundial.

Si bien esta idea de sociedad mundial se sigue lógicamente de los planteamientos de Luhmann, ella refleja una imagen algo formal y empíricamente poco empleable para dar cuenta de la interrelación entre el nivel operativo-estructural general de la sociedad mundial en formación, el local del Estado territorial y el regional en tanto agregación de espacios sociales con una distinguible afinidad institucional o semántica. De cualquier modo, entendido en ese nivel de generalidad, el concepto de sociedad mundial permite tres afirmaciones: (a) que a inicios del siglo XXI ya no es posible encontrar islas de socialidad (Fuchs, 2000): no existen regiones del globo para las cuales la coordinación bajo las exigencias de una comunicación sistémicamente diferenciada no tenga consecuencias importantes, en muchos casos decisivas, para los ordenamientos regionales y/o nacionales a lo largo del planeta (Stichweh, 2000; Mereminskaya y Mascareño, 2005); (b) que son estos ordenamientos regionales y nacionales los que permiten la variabilidad empírica de los diversos complejos sistémicos que caracterizan la sociedad moderna (Stichweh, 2004a, 2004b), y (c) que dada esta creciente universalidad de la 
sociedad mundial el análisis sociológico de cualquier contexto regional no puede ser llevado a cabo hoy con prescindencia de las referencias a tales condiciones estructurales y semánticas (Luhmann, 1997a, 1998; Luhmann/Di Giorgi, 1998). Tanto la creciente consolidación del proceso de diferenciación funcional de sistemas en el plano estructural como el progresivo predominio de un horizonte semántico universalista (Habermas, 2000; Habermas y Rawls, 1998; Rawls, 2002; Chernilo y Mascareño, 2005) exigen una mirada de la sociedad que intente acoplar el espacio geográfico en el que la observación se sitúa con procesos globales de carácter supranacional.

Para una aproximación más empírica a la observación del proceso de constitución de una sociedad mundial, los planos que deben ser vinculados son tres niveles emergentes relacionados entre sí por influencias ascendentes y descendentes: la diferenciación funcional de alcance global, la figura del Estado nacional como unidad territorial y la idea de región como espacio con una unidad institucional y semántica identificable. Que se trate de órdenes emergentes (Archer, 1997) significa: (a) que son niveles no sólo analítica sino ontológicamente distinguibles, es decir, que tienen modos de organización, distinciones y referencias sustantivas diferentes; (b) que cada uno de estos estratos muestra una alta autonomía operativa, es decir, sus formas estructurales y semánticas logran altos grados de reproducción interna, lo que les permite variar constantemente bajo parámetros conocidos, y (c) que gracias a su autonomía operativa estos órdenes emergentes muestran también alta interdependencia con los otros niveles, de modo que las transformaciones en un nivel constituyen irritaciones que pueden ser aceptadas o rechazadas por los otros, pero que en ningún caso les son indiferentes y tienen consecuencias recíprocas para sus formas de funcionamiento. Simplificado esquemáticamente, el modelo es como sigue: 
146 LOS DESAFÍOS DE LOS POSGRADOS EN CIENCIAS SOCIALES EN CHILE -

Teresa Matus, Aldo Mascareño, Adriana Kaulino

Tabla 1: Niveles de la sociedad mundial

\begin{tabular}{|l|l|l|l|}
\hline \multirow{2}{*}{} & \multicolumn{3}{|c|}{ Sociedad mundial } \\
\cline { 2 - 4 } & Nivel estatal nacional & \multicolumn{1}{|c|}{ Nivel regional } & \multicolumn{1}{|c|}{ Nivel global } \\
\hline Modo de organización & Estado/Nación & Institucionalidad regional & Diferenciación funcional \\
\hline Distinción central & Centro/periferia & Regional/local & Sistema/entorno \\
\hline Distinción secundaria & Inclusión/exclusión & Inclusión/exclusión & Inclusión/exclusión \\
\hline Referencia sustantiva & Territorio & Espacio & Comunicación \\
\hline
\end{tabular}

La diferenciación funcional, cuya radicalización puede rastrearse en el siglo XIX, supone la distinción de esferas operativas diferenciadas que contribuyen a la resolución de problemas sociales creados por la misma sociedad en el transcurso de su evolución. La política se ocupa principalmente del problema del orden, para lo cual traza decisiones colectivas vinculantes que afectan en forma directa a una población circunscrita a un determinado territorio (Luhmann, 2000). En el nivel sistémico global esta función vale para todo territorio, pero a la vez en todo territorio las decisiones tomadas son distintas. Mediante organizaciones como la escuela o la universidad, la educación se encarga de la selectividad pedagógica de los individuos. Esta función vale también para todo territorio, pero en todos ellos la selectividad se realiza de modos distintos y son precisamente esos territorios los que plantean exigencias diferenciadas a las distintas estructuras educativas regionales o territoriales (Luhmann y Schorr, 1979). Es decir, la diferenciación funcional no obliga a la unidad de decisiones, pero sí promueve la unidad de la función y la generación de instituciones de relativa homogeneidad en esos territorios: un poder ejecutivo, instituciones de administración, procedimientos eleccionarios e instituciones de control de decisiones.

De ello se derivan fuertes tensiones, en especial en el ámbito que interesa a esta investigación y que perfilan la distinción temáticoprofesional/disciplinar referida a la formación de posgrado. Puesto que al Estado territorial le resulta difícil aceptar las presiones descendentes de la diferenciación funcional de carácter global, en tanto ellas provienen de un centro excéntrico y muchas veces indefinido, el Estado, como centro, busca controlar su periferia intentando establecer límites positivos y negativos a la operación de sistemas en el marco de 
su territorio. Por límites positivos se entienden definiciones acerca de rendimientos políticamente relevantes de esferas no políticas: sistemas de seguridad social, estrategias universales de protección de salud, desarrollo de determinadas esferas de producción en la economía y formación de capital humano en la educación; por límites negativos, límites regulatorios, en general jurídicos, de operación en cada uno de los distintos sistemas, que no indican qué se debe hacer, sino que plantean un marco de condiciones que deja abierto un espacio de autonomía para la operación de las distintas esferas.

En el marco de los límites negativos, los sistemas pueden operar con autonomía y acoplarse a los requerimientos y características propias de sus espacios supranacionales -esto es, globales- de operación. En lo que nos interesa, el espacio de posgrados en ciencias sociales, los mecanismos de formación se conectan estrechamente con las teorías y métodos de las esferas disciplinares de nivel global. Esto conduce a un énfasis en la formación disciplinar en los posgrados en el nivel territorial. Los límites positivos, en tanto, puesto que implican la definición de intereses temático-profesionales generalmente asociados a expectativas de desarrollo nacional impulsadas por la política, son transversales a los intereses disciplinares autónomos de la formación de posgrado. Cuando el énfasis está puesto en estos últimos, se generan programas de formación de posgrado en ciencias sociales de carácter temático-profesional; cuando está puesto en límites positivos, se abre la posibilidad de desarrollo de programas de carácter disciplinar.

Ambos no son, por cierto, excluyentes. Por ello, la distinción temático-profesional/disciplinar oscila permanentemente y genera énfasis diferenciados en los programas de estudio. Lo que se debe tener claro, por ahora, es que esta distinción, más que una decisión personal o institucional por uno u otro tipo de formación de posgrado, expresa la vinculación de los planos territorial, regional y global de la sociedad mundial y las influencias diferenciadas que estos espacios tienen en la formación de posgrado en Chile: para los posgrados temáticos-profesionales la referencia territorial y regional es la más 
relevante; para los disciplinares la referencia global especializada en el campo de conocimientos específico (psicología, sociología, trabajo social, antropología, ciencia política) es la central.

El nivel regional, cuya referencia sustantiva es el espacio y su modo de ordenamiento lo constituye una organización regional (generalmente de Estados, pero también de actores y redes de instituciones), opera de manera interna mediante una distinción entre región y localidad, en la cual la región es un orden emergente basado en la asociación de localidades múltiples, cuya unidad define un espacio regional (Stichweh, 2000) que siempre muestra un nivel mayor o menor de institucionalización. El nivel regional opera sobre un sustrato más abstracto que el Estado nacional: el espacio, no directamente el territorio; aunque tampoco se constituye con pura referencia al tipo de comunicación específico, como lo hace la diferenciación funcional. Ciertamente, el espacio constituye una indicación en la comunicación que se transforma en una semántica estabilizada en los arreglos institucionales de aquellos que se consideran miembros de la región (aunque también para los que están excluidos de ella). Pero esa indicación semántica remite, a la vez, a actores situados en territorios específicos, los que por su mayor o menor distancia geográfica, precisan de una denominación más general que los incluya: la de espacio regional (Stichweh, 2003, 2005).

Tanto la región espacial como el territorio estatal nacional constituyen, por tanto, formas de realización empírica de la diferenciación funcional global y, a la vez, contribuyen a ella y la tensionan con demandas de adecuación o inadecuación (inclusión/ exclusión) de los modos de operación sistémicos a las condiciones espaciales o territoriales.

Una distinción relevante cruza también los tres niveles de la sociedad mundial: la distinción inclusión/exclusión. Por medio de ella, estos niveles emergentes ponen atención en la participación/ no-participación de personas o unidades mayores (organizaciones, 
Estados) en sus respectivos rendimientos. El nivel global de la diferenciación funcional no sólo observa la inclusión o exclusión de personas en rendimientos políticos, económicos o educativos, sino que también, al poner atención al nivel espacial regional, puede indicar que ciertas regiones están más excluidas que otras de los rendimientos que ofrecen los sistemas y calificarlas como regiones subdesarrolladas; o si la mirada es puesta en el territorio, como Estados subdesarrollados. Este es el modelo de Wallerstein del sistema mundial (2004) o, más radicalmente, el modelo desarrollista desplegado por la Comisión Económica para América Latina y el Caribe (CEPAL) en los años cincuenta del siglo pasado (Larraín, 1989), que extendió la distinción territorial centro/periferia a la observación de la sociedad mundo. La subcomplejidad de estos modelos para la descripción actual de la sociedad mundial es clara. Atribuyen demasiada relevancia a la dimensión territorial (la esencializan) y subvaloran las constelaciones supranacionales de sistemas diferenciados y semánticas crecientemente universales de la sociedad mundial. No logran observar la alta inclusión que existe en territorios o regiones de la sociedad mundial.

En el campo de la educación, el mejoramiento de las condiciones de inclusión locales (en el nivel estatal) ha conducido a la formación de redes regionales de escuelas que comparten sus experiencias, innovan en mecanismos pedagógicos y generan una presión descendente sobre sus Estados por modificaciones regulatorias o financieras (Smith y Wohlstetter, 2001; Veugelers y Zijlstra, 2002). En el nivel superior de la diferenciación funcional global, ello conduce a reestabilizaciones en los modos de acoplamiento estructural entre educación, política, economía y sistema jurídico, las que quedan a disposición de otros espacios regionales y territorios para nuevas modificaciones.

El movimiento ascendente y descendente entre estos niveles es precisamente lo que queremos observar ahora a propósito de la formación de posgrado en ciencias sociales en Chile. Como ha sido planteado, realizamos esto mediante la distinción temáticoprofesional/disciplinar, en tanto ella permite observar, por un lado, 
150 LOS DESAFÍOS DE LOS POSGRADOS EN CIENCIAS SOCIALES EN CHILE -

Teresa Matus, Aldo Mascareño, Adriana Kaulino

cómo la estructura nacional de posgrado instala distinciones e intereses territoriales o regionales en un contexto global y, por otro, cómo los contextos globales de las diversas disciplinas colocan sus temas e intereses en los espacios territoriales y regionales.

\section{Complejidad de la sociedad mundial y la formación de posgrado}

La existencia de una sociedad mundial no supone su homogeneidad. Ya desde un punto de vista empírico esa posición se torna insostenible. Las regiones y territorios desarrollan particularidades estructurales y semánticas que impiden pensar en una sociedad mundial como un espacio homogéneo de comunicación. Por otro lado, el despliegue de sistemas funcionales en el plano global promueve la observación de la sociedad desde la perspectiva de cada sistema, de modo que esa única sociedad se desagrega y se interpreta de manera heterogénea, lo que conduce a una virtualización de la sociedad y a su lectura como diferencia o como unitas multiplex.

Los conceptos de "complejidad" y "emergencia" deben entenderse como mutuamente referidos. La complejidad es condición de posibilidad de la emergencia. Cuando un conjunto de elementos ya no puede ser relacionado uno a uno en un mismo momento entonces se habla de complejidad. Desde el punto de vista de los individuos, complejidad implica la imposibilidad de vivenciar múltiples experiencias y realizar variadas acciones. La complejidad social, en este sentido, es producto de la diferenciación de sistemas en la sociedad mundial: diversas interacciones, organizaciones y esferas funcionales, como la educación, la política, el derecho, la religión (Luhmann, 1984). A la vez, esta diferenciación de sistemas es producto del proceso de evolución social que especializa distintos ámbitos de sentido y los orienta a la resolución o al tratamiento de problemas sociales que tienen lugar y son producidos por esos mismos ámbitos de sentido y por sus acoplamientos con otros ámbitos (Luhmann, 2007). Así, la necesidad de una formación de posgrado en ciencias sociales es tanto un producto de la existencia de un sistema de organizaciones 
universitarias de alcance mundial, de la diferenciación de disciplinas sociales (antropología, sociología, trabajo social, psicología, ciencia política), como también de las necesidades de espacios funcionales como la economía, el derecho, la salud y la política, que requieren la inclusión de individuos manejando las operaciones centrales y posibilitando la reproducción de esas esferas.

En tal sentido, la denominación "ciencias sociales", que caracteriza genéricamente el ámbito de estudios de posgrado en este campo, es una fórmula que reduce la contingencia de las diversas especializaciones en las ciencias sociales. La semántica de las ciencias sociales remite a la unidad la diferencia de distintos campos cuyos elementos ya no pueden ser relacionados uno-a-uno en un programa de estudios. Por ello, programas que pretenden ser de formación de posgrado en ciencias sociales deben inevitablemente no serlo. Es decir, deben reducir la complejidad de la diferenciación y especialización de las ciencias sociales y seleccionar algunos elementos de ella para vincularlos entre sí en un programa específico. En tal sentido, estos programas se presentan como imposibilidad y, aunque aquella sea su autodescripción, sólo pueden abarcar una dimensión mínima de la complejidad de las ciencias sociales.

De este modo, complejidad siempre implica contingencia de múltiples opciones y éstas siempre suponen una selección, pues no se pueden seleccionar todas las opciones al mismo tiempo (Luhmann, 2007). La distinción temático-profesional/disciplinar referida a los posgrados en ciencias sociales opera así como una diferencia directriz (Luhmann, 1984) que permite seleccionar un punto de observación para el diseño de un programa de posgrado en esta área; o se orienta la observación hacia temas específicos que ponen el énfasis en dimensiones territoriales o regionales, o hacia los métodos y teorías de la disciplina, con lo que se pone énfasis en el espacio global de cada ciencia.

Puesto que la complejidad supone contingencia y la contingencia selección, los elementos seleccionados en la formación 
de un posgrado tienen la pretensión de operar como sistema. Se definen procedimientos de evaluación, selección y egreso, mallas curriculares, criterios de costos, perfiles de postulantes, perfiles de egresados y objetivos pedagógicos. Es decir, se establecen elementos y relaciones entre éstos que reducen la complejidad de las múltiples operaciones posibles en la formación de posgrado en ciencias sociales. No obstante, esos mismos elementos y relaciones constituyen, en sí mismos, un sistema complejo que, en tanto sistema, opera en un nivel emergente, es decir, no puede reducirse a la suma de sus partes: es más que esa suma. Un orden complejo es un orden emergente, en tanto que el intento de descripción de ese orden escapa a la simple representación de sus componentes y debe considerar la totalidad de sus relaciones y la novedad que se deriva de ellas. Esta novedad de la emergencia de un programa es lo que se puede captar mediante el concepto de "sentido".

El sentido es la forma de procesamiento de la complejidad que ejercen los sistemas sociales que operan bajo la forma del sentido. Es resultado de la selección de posibilidades en un mundo complejo, las operaciones actualizadas por las cuales el sistema conduce su propio funcionamiento en la dirección que el sentido expresa (Luhmann, 1984). Una primera distinción en el sentido, en el caso de los posgrados en ciencias sociales, viene dada por el modo en que la diferencia directriz temático-profesional/disciplinar conecta a esos programas con los contextos territoriales, regionales y globales de la sociedad mundial, como se vio en la sección anterior. Pero el sentido permite una caracterización mayor en tanto se reconoce la problemática de sus dimensiones.

El sentido puede ser reconocido bajo la operación de tres dimensiones: objetual, social y temporal. La primera permite distinguir entre 'esto' y lo 'otro', es decir, se trata de un mecanismo que permite construir objetos de referencia por medio de la observación y la descripción. La propia diferencia directriz temático-profesional/ disciplinar opera eficientemente para esta construcción de sentido objetual. En el campo temático, permite la definición de múltiples 
opciones para el diseño de un programa de posgrado. En tal campo, las posibilidades son infinitas y sólo pueden estar constructivamente restringidas por la operación de las otras dos dimensiones del sentido: la social (iexiste público para un determinado tema?) y la temporal (ies posible realizarlo en un tiempo prudente?). En el campo disciplinar, la dimensión objetual parte desde la reducción de sentido hecha evolutivamente por la constitución de disciplinas: en principio, un posgrado en sociología no actualiza la posibilidad de ser un posgrado en psicología. Esto elimina contingencia para la formación de posgrados disciplinares, pero la abre internamente a la contingencia y complejidad de la disciplina de que se trate: la abre a énfasis en distintas corrientes teóricas disciplinares o a distintos métodos de investigación y su uso en esos campos. Las posibilidades son también múltiples en este aspecto e, igualmente, la dimensión social y la temporal operan como restrictores de los posibles temas disciplinares.

La dimensión social del sentido, en tanto, remite a la relación entre alter y ego, sean estos sistemas de cualquier tipo. Mediante la dimensión social del sentido es posible reconocer que en el mundo existe un otro cuya capacidad de observación es idéntica a la del observador pero que, a pesar de ser idéntica y tener las mismas facultades a su disposición, observa de otra manera. De esta dimensión deriva la forma de la doble contingencia de la comunicación en la sociedad y de ello se deduce precisamente su complejidad, en tanto cada uno vivencia y actúa en el mundo de la manera en que cada uno puede hacerlo y no de otra. Esta dimensión es relevante en la formación de posgrado, tanto por los procesos pedagógicos que tienen lugar, pues ellos siempre suponen la relación entre alter y ego, como también por las observaciones de cada programa sobre su entorno relevante, tanto en la búsqueda de una especificidad propia como programa de posgrado -que se logra por la dimensión objetual: este tema, estas teorías- como en la búsqueda de los públicos a los cuales se quiere llegar con el sentido del programa.

Finalmente, la dimensión temporal se relaciona con la idea de horizonte. Todo el pasado y el futuro se funden en la idea de horizonte, 
independientemente de que sea representado cronológica o en forma lineal (Luhmann, 1984). Como todo horizonte, la dimensión temporal del sentido es siempre difusa, pues el horizonte se mueve en tanto se está en uno u otro momento. Por medio de diversos mecanismos, esos horizontes se pueden fijar, pero puesto que los tiempos se fijan en el sentido, este mismo los puede cambiar. No hay un principio ni un plazo final.

En el caso de la formación de posgrado, esta dimensión es relevante por varios aspectos. Permite fijar un horizonte en el cual los programas se cumplen, procedimientos para cumplir los objetivos cuando los programas no se cumplen y sanciones cuando el programa no se cumplió, es decir, cuando la falta se evalúa como pasado irreversible. La dimensión temporal permite también la proyección de la transformación del propio programa: se puede iniciar con una malla curricular prototípica e irla adecuando al tema o a la disciplina en el futuro; permite igualmente incorporar nuevos conocimientos temáticos o nuevos avances disciplinares en la formación, así como dejar atrás los conocimientos obsoletos e incluso introducir cambios para criticarlos. Sobre la base de las tres dimensiones del sentido es posible definir un campo de referencias de los programas de posgrado en ciencias sociales con relación a lo observado en la sección anterior. Esquemáticamente este campo puede ser diseñado del modo siguiente:

Tabla 2: Campo de referencias según orientación temático-profesional/disciplinar

\begin{tabular}{|c|c|c|c|}
\hline & \multicolumn{2}{|c|}{ Temático-profesional } & Disciplinar \\
\hline & Territorial & Regional & Global \\
\hline Objetual & Desarrollo nacional & Desarrollo nacional-regional & Investigación disciplinar \\
\hline Social & $\begin{array}{c}\text { Otros programas } \\
\text { nacionales / Público } \\
\text { nacional }\end{array}$ & $\begin{array}{c}\text { Otros programas nacionales- } \\
\text { regionales / Público nacional- } \\
\text { regional }\end{array}$ & $\begin{array}{c}\text { Otras disciplinas/ Público } \\
\text { disciplinar }\end{array}$ \\
\hline Temporal & Horizontes políticos & Horizontes políticos & Horizonte disciplinar \\
\hline
\end{tabular}

Varias conclusiones preliminares pueden ser extraídas de esta tabla. Las presentamos primero en términos formales para, en la siguiente sección, darles sustento empírico a partir de los hallazgos de la investigación: 
- La distinción directriz temático-profesional/disciplinar permite diferenciar las orientaciones de referencia básica de los posgrados en ciencias sociales en Chile. Los temáticos remiten al territorio o a la región; los disciplinares a la investigación y profundización en el campo disciplinar de que se trate. A estos últimos, entonces, se los puede considerar como posgrados con un interés supranacional.

- En la dimensión objetual comienzan a especificarse estas orientaciones fundamentales. La referencia básica de los posgrados temáticos es el desarrollo nacional o regional. Esto se observa en la definición de temáticas orientadas a las políticas públicas en términos amplios, es decir, en esta dimensión los posgrados temático-profesionales buscan responder a necesidades predefinidas políticamente.

- En tanto los posgrados disciplinares se orientan a la profundización y especialización en el campo disciplinar, su referencia central no es política sino científica. Los intereses básicos vienen guiados por la reflexión sobre la propia disciplina con un horizonte espacial que no atribuye relevancia a fronteras nacionales.

- Esta referencia hacia intereses políticamente definidos o propiamente científicos se expresa en la dimensión social tanto en otros programas que se definen como referentes (de competencia o de complementación) como en los públicos hacia los cuales los programas se orientan.

- La dimensión objetual también deja su rastro en la dimensión temporal. Si las orientaciones fundamentales son políticas en un caso y científico-disciplinares en otro, entonces las expectativas temporales de cada programa se definen en los horizontes temporales de esos ámbitos, por ejemplo, en la definición de los horarios presenciales o en las perspectivas de inclusión de sus miembros.

- Una tesis general se deriva también de estas observaciones: es la tendencia hacia la desdiferenciación de intereses del campo propiamente científico por orientaciones de tipo político. Ello produce una consecuencia altamente problemática en la formación de posgrado asociada a los límites y a la constitución de disciplinas fuertes que permitan la investigación de temáticas sociales en 
múltiples sentidos. Si la referencia primaria, sin embargo, es temática-profesional y la referencia disciplinar es subvalorada, los intereses políticos inmediatos se constituyen en obstáculos para el desarrollo disciplinar en Chile. Asimismo, resulta problemático observar un criterio homogeneizante en las políticas de acreditación de posgrados, las cuales son inmunes a esta distinción.

Sobre la base de estos puntos queremos ahora desplegar el análisis de los elementos empíricos de esta investigación. Con ello se pretende dar cuenta de las tesis recién esbozadas y mostrar los alcances de la distinción directriz temático-profesional/disciplinar que orienta la operación de los programas de posgrado en ciencias sociales en Chile.

\section{Posgrados en ciencias sociales en Chile: la diferencia directriz y sus consecuencias}

Desde las premisas antes descritas se puntualizan empíricamente algunas conclusiones. Para esto nos remitimos tanto a las representaciones a través de las cuales los programas de posgrado se describen a sí mismos como a las descripciones que observadores expertos hacen de ellos en términos generales. Si bien estas conclusiones son referidas puntualmente, a todas ellas subyace una hipótesis general basada en las premisas teóricas descritas.

1. Hipótesis sobre la diferencia directriz temáticoprofesional/disciplinar

La diferencia directriz temático-profesional/disciplinar es la distinción rectora del diseño y estructura de posgrados en ciencias sociales en Chile. Esto implica que:

- Mediante ella es posible analizar las diferencias y similitudes de los posgrados en ciencias sociales en Chile.

- Las diferencias y similitudes se derivan de la oscilación de la diferencia, es decir, de los énfasis que cada programa ponga en uno u otro lado de la distinción. 
- Si bien, producto de la oscilación de esa diferencia, es posible distinguir en términos amplios entre posgrados temáticosprofesionales y disciplinares, ello no supone que los programas temáticos no conserven elementos disciplinares o viceversa.

- Esta oscilación de la diferencia directriz depende de cuánto influyan lógicas políticas (orientación a lo temático) o científicas (orientación a lo disciplinar) en el diseño de los programas de posgrado.

- La oscilación de la diferencia directriz supone la introducción de elementos disciplinares en programas temáticos y de elementos temáticos en programas disciplinares. En los primeros se trata de herramientas metodológicas entendidas en términos de técnicas subordinadas al objetivo temático; en los segundos, de temáticas disciplinares.

2. Trasfondo de la diferencia directriz: desdiferenciación política

La diferencia directriz temático-profesional/disciplinar que opera para los posgrados tiene su sustrato en lo que puede denominarse un predominio de la comunicación política en la sociedad chilena. Esto no sólo afecta al área de los posgrados, sino que, en general, al sistema educativo y también a otros espacios funcionales como el derecho, los medios de comunicación y la economía. Esta constelación problemática puede denominarse desdiferenciación sistémica (Mascareño, 2007). Aplicada esta conceptualización a la temática de los posgrados en ciencias sociales en Chile, se obtienen los siguientes resultados:

- Puesto que en los niveles territoriales y regionales de la sociedad mundial predominan las semánticas de integración política, sea nacional o regional, el sistema universitario en general es puesto ante una presión de contribución al desarrollo nacional y a la integración regional. Esto es particularmente fuerte en países y regiones con una alta desigualdad social y con temáticas no resueltas en términos de desarrollo económico.

- En estos niveles, las semánticas de integración social y de contribución al desarrollo, de origen fundamentalmente político, 
generan altos obstáculos al despliegue científico de disciplinas (Chernilo/Mascareño, 2005), que se desarrollan en una dimensión supranacional, responden a lógicas autónomas de operación del sistema científico y plantean exigencias que son propias para el desarrollo de sus conocimientos, independientes de su utilidad política.

- Los posgrados de orientación temática-profesional han incorporado estas pretensiones políticas a través de sus orientaciones programáticas, es decir, ha tenido lugar una desdiferenciación de sus operaciones.

- Los posgrados de orientación disciplinar presentan un acoplamiento estrecho con los intereses y constelaciones problemáticas de la propia disciplina y rechazan las limitaciones y constricciones de carácter político nacional o regional.

\section{Los posgrados de ciencias sociales en números}

Sobre la base de la diferencia directriz temático-profesional/disciplinar la investigación ha construido la siguiente tabla:

Tabla 3: Número de doctorados en ciencias sociales según distinción temático-profesional/ disciplinar

\begin{tabular}{|l|c|c|}
\hline & Temático-profesional & Disciplinar \\
\hline Doctorados & & 5 \\
\hline Psicología & - & 2 \\
\hline Sociología & - & $1^{*}$ \\
\hline Antropología & - & 0 \\
\hline Trabajo Social & - & 1 \\
\hline Ciencia política & - & - \\
\hline Ciencias sociales & $4^{* *}$ & . \\
\hline
\end{tabular}

*El doctorado en Antropología es conjunto entre la Universidad de Tarapacá y la Universidad Católica del Norte.

** Como la investigación se acota a ciencias sociales, no se ha considerado el doctorado temático en Filosofía, mención Epistemología de las Ciencias Sociales, en la Universidad de Chile, por estar inserto más bien en el área de Humanidades y Filosofía.

Según los cálculos más optimistas, el panorama en términos de doctorados muestra, en realidad, un cuadro incipiente. Claramente, 
psicología es la disciplina más consolidada en las ciencias sociales en Chile, con cinco doctorados y dos de ellos ya acreditados. Luego, hay una consolidación regional en el caso de antropología con el doctorado en el norte del país. Asimismo, los doctorados en sociología, aunque de reciente creación, cuentan ya con un programa acreditado. Los doctorados en Ciencias Humanas, mención discurso y cultura de la Universidad Austral, en Estudios Latinoamericanos de la Universidad de Chile y en Estudios Latinoamericanos de la Universidad de Santiago completan el índice de los acreditados. De allí que ciencia política sea claramente emergente y trabajo social aún no cuente con doctorado en el país. Ahora bien, si analizamos los antecedentes recopilados en términos de magíster, ello confirma la tendencia antes señalada: existe una clara preeminencia de psicología con 46 programas. A ella le siguen muy de lejos trabajo social y ciencia política, con cuatro cada uno, antropología con 3 y sociología con otros 3 programas. Es decir, de 60 programas disciplinarios, el 77,9\% corresponden a psicología.

Tabla 4. Diferenciación de magísteres de ciencias sociales en Chile

\begin{tabular}{|l|c|}
\hline 2.1 Magíster disciplinario & No Total \\
\hline 2.1.1 Psicología & 46 \\
\hline 2.1.2 Sociología & 3 \\
\hline 2.1.3 Antropología & 3 \\
\hline 2.1.4 Trabajo social & 4 \\
\hline 2.1.5 Ciencia política & 4 \\
\hline 2.2 Magíster temáticos en el área de las ciencias sociales & $\mathrm{N}^{\circ}$ Total \\
\hline 2.3 Magíster en ciencias sociales & $\mathrm{N}^{\circ}$ Total \\
\hline Total de magísteres en ciencias sociales en Chile & 9 \\
\hline
\end{tabular}

Sobre la base de esta información y de las argumentaciones, es posible apuntar los siguientes elementos:

- Si los doctorados pueden ser entendidos como el nivel más alto en la formación disciplinar, es claro que en Chile, en términos generales, el desarrollo disciplinar de las ciencias sociales es todavía incipiente.

- La observación anterior se apoya también en el alto número de posgrados temáticos en el área de la denominación genérica 
"ciencias sociales" comparativamente, tanto en el área de doctorados como en la de magíster.

- Ciertamente, el indicador de número de posgrados disciplinares no es el único que puede aportar al tema de la constitución de disciplinas en Chile, aunque sí se trata de uno particularmente relevante, pues evidencia los puntos siguientes:

* Los posgrados disciplinares permiten la formación en investigación disciplinar, es decir, con los métodos y teorías propios de cada disciplina.

* Estos posgrados ponen en relación la academia nacional con las discusiones académicas supranacionales de la disciplina, es decir, vinculan directamente el espacio académico local con el espacio global disciplinar por medio de discusiones, redes e intercambios.

* Cualquier pretensión de investigación de carácter transdisciplinar -semántica con la que se justifican los posgrados temáticos- no puede sino estar basada sobre disciplinas fuertes. Si éstas no existen, la investigación temática es mucho más proclive a someterse a intereses extraacadémicos, principalmente políticos, aunque también económico-corporativos.

\section{Programas según nivel de acreditación}

Tabla 5

\begin{tabular}{|c|c|c|c|c|}
\hline Disciplinas/Nivel de acreditación de posgrados & \multicolumn{2}{|c|}{ Acreditado } & \multicolumn{2}{|c|}{ No Acreditado } \\
\hline Psicología & $\begin{array}{l}\text { Doctorado } \\
\text { Magíster }\end{array}$ & $\begin{array}{l}=2 \\
=7\end{array}$ & $\begin{array}{l}\text { Doctorado } \\
\text { Magíster }\end{array}$ & $\begin{array}{l}=3 \\
=39\end{array}$ \\
\hline Sociología & $\begin{array}{l}\text { Doctorado } \\
\text { Magíster }\end{array}$ & $\begin{array}{l}=1 \\
=1\end{array}$ & $\begin{array}{l}\text { Doctorado } \\
\text { Magíster }\end{array}$ & $\begin{array}{l}=1 \\
=2\end{array}$ \\
\hline Antropología & $\begin{array}{l}\text { Doctorado } \\
\text { Magíster }\end{array}$ & $\begin{array}{l}=1 \\
=2\end{array}$ & $\begin{array}{l}\text { Doctorado } \\
\text { Magíster }\end{array}$ & $\begin{array}{l}=0 \\
=1\end{array}$ \\
\hline Trabajo social & Magíster & $=2$ & Magíster & $=2$ \\
\hline Ciencia política & Magíster & $=1$ & $\begin{array}{l}\text { Doctorado } \\
\text { Magíster }\end{array}$ & $\begin{array}{l}=1 \\
=3\end{array}$ \\
\hline Ciencias sociales & Magíster & $=1$ & Magíster & $=8$ \\
\hline Temáticos & $\begin{array}{l}\text { Doctorado } \\
\text { Magíster }\end{array}$ & $\begin{array}{l}=3 \\
=2\end{array}$ & $\begin{array}{l}\text { Doctorado } \\
\text { Magíster }\end{array}$ & $\begin{array}{l}=1 \\
=23\end{array}$ \\
\hline Totales según número de programas & $\begin{array}{l}\text { Doctorado } \\
\text { Magíster }\end{array}$ & $\begin{array}{l}=7 \\
=16\end{array}$ & $\begin{array}{l}\text { Doctorado } \\
\text { Magíster }\end{array}$ & $\begin{array}{l}=6 \\
=78\end{array}$ \\
\hline
\end{tabular}


Podemos afirmar que existe un bajo número de programas acreditados, tanto de doctorado (sólo un 53\%) como de Magíster (de un total de 94, sólo 19 están acreditados por la CONAP, es decir, un $20,2 \%)$.

\section{Nivel de transparencia en la información}

Tabla 6

\begin{tabular}{|l|c|c|c|}
\hline Disciplinas/Calidad de la información en la web & Mínima & Incompleta & Completa \\
\hline Psicología & 4 & 47 & 0 \\
\hline Sociología & 1 & 3 & 0 \\
\hline Antropología & 0 & 4 & 0 \\
\hline Trabajo social & 0 & 4 & 0 \\
\hline Ciencia política & 0 & 4 & 0 \\
\hline Ciencias sociales & 7 & 2 & 0 \\
\hline Temáticos & 4 & 23 & 0 \\
\hline Totales según número de programas & 16 & 87 & 0 \\
\hline
\end{tabular}

En general, la información que se publicita en las páginas web de las carreras o programas que dictan el posgrado es incompleta. Salvo excepciones, no es posible saber cuáles son las líneas de investigación y las principales publicaciones de los académicos, los títulos de las tesis presentadas o el número de estudiantes que han obtenido el grado. En fin, en materia de accountability, falta mucho para tener un acceso universal y democrático a indicadores generales de los posgrados en ciencias sociales en Chile.

\section{La diferenciación de la psicología como disciplina}

A juzgar por los datos presentados, la psicología destaca por sobre las otras ciencias sociales en el área de los posgrados como una disciplina con un fuerte desarrollo académico. Con 46 magísteres y cinco doctorados, la estructura de posgrados en esta disciplina parece estar alcanzando madurez y consolidación en la universidad chilena.

La diferenciación de la psicología como una disciplina con alta presencia en posgrado puede explicarse, de acuerdo con los observadores expertos entrevistados, por el "bache académico" que 
se produjo en el periodo de la dictadura en Chile y que eliminó la contingencia disciplinar en las ciencias sociales. Esto significó dos cosas: a) una hiperprofesionalización que las mantuviera lejos de las cuestiones doctrinarias a las cuales se les asociaba en los años sesenta, y b) la instrumentalización y descolectivización del análisis social. Con ello, la psicología (como técnica, especialmente la psicología clínica, y, como teoría, sobre todo su foco en el individuo) aparece como una materia neutra a problemas políticos o ideológicos y se desarrolla entonces disciplinarmente. Por otra parte, para perfilarse en ese marco, ha tenido que focalizar sus publicaciones en términos de artículos ISI ${ }^{1}$.

Para las otras ciencias sociales la alternativa estuvo constituida por: a) una neutralización de su campo teórico, b) una sobrevaloración de los instrumentales metodológicos y c) una consecuente orientación a la investigación temática. Esto recién parece cambiar hacia mediados de los años noventa, con una nueva generación de investigadores jóvenes en otras áreas de las ciencias sociales, cuya formación se realiza principalmente en centros de investigación extranjeros y que se ve reflejada en el incipiente desarrollo de otros programas de posgrado de carácter disciplinar.

Como modelo de la orientación disciplinar en psicología puede considerarse el doctorado en Psicología de la Pontificia Universidad Católica de Chile (PUC). El programa, acreditado por CONAP, se autodescribe orientado a la investigación y la docencia universitaria en los campos de la salud mental y la psicoterapia, con un especial énfasis en la disminución "de la brecha entre clínica e investigación a través de la formación en conocimiento científico referido a modalidades terapéuticas y su efectividad" (pág. web). Así también, el programa busca establecer nexos con otros espacios donde se desarrolla la disciplina psicológica, captando estudiantes extranjeros y esperando lograr este nexo no de un modo temático, sino "a través de la docencia y la productividad en investigación del cuerpo académico" (pág. web).

\footnotetext{
1 Base de datos con revistas seleccionadas del Institute for Scientific Information (ISI).
} 
Claramente, el desarrollo disciplinar se entiende a partir de la referencia a la disciplina y a lo central de ella: el desarrollo de conocimientos por medio de la investigación científica y su posterior transmisión y formación de nuevos científicos a través de las actividades de docencia académica. Interesante es también en este caso la constitución de un cuerpo de profesores que se forma principalmente en universidades europeas de fuerte desarrollo disciplinar en Alemania, Reino Unido y Estados Unidos.

\section{El problema político-financiero y la escisión de teoría y método}

Desde los observadores expertos puede también derivarse una razón para la constitución de posgrados de carácter temático: la visualización de los posgrados como instrumentos financieros para la estabilidad de la organización universitaria. Producto de la profesionalización y desustantivación de las ciencias sociales en el periodo de la dictadura, en los años noventa se constituye un escenario altamente tecnificado para los posgrados en esta área. Si a esto se suma la demanda política del periodo democrático por unas ciencias sociales que contribuyan con sus conocimientos a la resolución de la "agenda social", entonces se tiene un resultado específico: los posgrados en ciencias sociales remiten principalmente a una dimensión temática para la cual las técnicas, pero no las teorías disciplinarias, son relevantes. En el ámbito disciplinar, esto representa una escisión de teoría y método.

Ninguna disciplina piensa y desarrolla sus métodos de investigación con independencia de sus cuerpos teóricos. Las teorías constituyen la argumentación sustantiva y académica del por qué determinados métodos en determinadas disciplinas son necesarios o, incluso, imprescindibles. Si se separan teoría y método, el método se constituye en una forma procedimental desligada de sentido, cuyos resultados pierden prestancia académica y pueden ser empleados para finalidades de otro tipo, por ejemplo, políticas.

La postergación de las ciencias sociales en el periodo de la dictadura puede ser vista a través de este desacoplamiento entre 
teoría y método, y permite observar también la vinculación del método con los intereses temáticos de la agenda sociopolítica y la formación de posgrados temáticos en ciencias sociales. Éstos tienen como referente la agenda político-social y emplean como instrumento los métodos de distintas disciplinas desacoplados de sus sustratos teóricos. El resultado es que con esos métodos se puede probar y realizar lo que el interés sociopolítico indique. En la semántica con que expertos describen la situación de los posgrados en Chile, a esto puede llamársele "profesionalización". En estas páginas lo hemos llamado "desdiferenciación": política de las disciplinas científicas de las ciencias sociales.

La dimensión económica de la profesionalización se vincula con las presiones que en los distintos campos de desarrollo del mercado se vuelven sobre los individuos para obtener calificaciones. Se establece con ello una demanda profesional de individuos sin interés académico, sino básicamente con un interés asociado a la obtención de un grado académico que permita un mejor posicionamiento estratégico en el espacio de trabajo, sea en términos de habilitación o de poder. Ello genera incentivos a las organizaciones universitarias para la reproducción de posgrados temáticos antes que disciplinares. Si a esto se suma la apertura de estas organizaciones a las operaciones de mercado, se tiene un panorama en que cada unidad académica de una universidad tiene libertad para desarrollar programas de posgrado en tanto puedan ser autofinanciados. Puesto que los programas temáticos tienen mucha más probabilidad de autofinanciamiento que los disciplinares, todos los incentivos del sistema están puestos en la generación de este tipo de programas. Lo que nuevamente conduce a un debilitamiento de las disciplinas al interior de las universidades, o a la ilusión de creación de interdisciplinariedad por medio de estos programas temáticos sin que existan disciplinas fuertes de fondo. 


\section{La contribución de los posgrados a la constitución disciplinar}

Donde no hay escisión entre teoría y método es en la formación de posgrado con orientación disciplinar. La escisión teoría/método, que tiene lugar en el ámbito de los posgrados temáticos, es reunida radicalmente en los que se autodescriben como formación disciplinar. En los primeros, el método adquiere la función de "caja de herramientas" que se pone a disposición de intereses temáticos, en general definidos políticamente (políticas públicas) o por los intereses profesionales de la demanda; en los disciplinares, se relaciona estrechamente con el desarrollo teórico en el objetivo de la investigación académica.

La contribución de los posgrados a la formación disciplinar es clara en el caso chileno de psicología, como ha quedado demostrado anteriormente. En el caso de la antropología, el desarrollo de una dimensión de investigación y análisis logró fructificar hace ya años en el doctorado conjunto entre la Universidad Católica del Norte y la Universidad de Tarapacá. Sin embargo, en los últimos años la sociología, la ciencia política y el trabajo social han comenzado a desarrollar una fuerte asociación entre formación de posgrado y acoplamiento con los respectivos espacios de disciplinas. El doctorado en Sociología de la Universidad Alberto Hurtado es un buen ejemplo de ello: no aparece motivado por una necesidad de expansión financiera, no apela a un mercado profesionalmente orientado ni tiene una orientación profesionalizante entre sus profesores. El fuerte de este programa es la investigación sociológica y se caracteriza por la múltiple inserción internacional de sus académicos. Asimismo, un nivel claro de enriquecimiento disciplinar lo constituye la próxima apertura del doctorado en Sociología de la PUC. Con más de una década en la dictación de su programa de magíster, cuenta con un cuerpo académico consistente, con líneas de investigación específicas, lo que conforma una potencialidad innegable para la disciplina. Por otra parte, a los programas de magíster existentes en ciencia política se acaba de sumar el doctorado en esa disciplina que abrirá la PUC en 2008. 
En el caso del magíster en Trabajo Social de la PUC -cuyo proceso de constitución disciplinar es siempre complejo, por su origen más bien asociado a programas sociales, en el marco de la acción de gobierno-, hay un interesante perfilamiento del trabajo social como disciplina especializada en procesos de intervención social. El programa se autodescribe como "profundización de conocimiento", como "desarrollo de competencias avanzadas en el ámbito de la intervención y la investigación social aplicada" (pág. web). Por otro lado, también cumple con una característica que, ya queda claro, es central para el desarrollo de programas disciplinares: la inserción de sus docentes en redes supranacionales de desarrollo disciplinar académico. Aún así, el trabajo social en Chile presenta una gran barrera para el aumento de sus investigaciones: no constituye grupo disciplinar en el Fondo de Desarrollo de Ciencia y Tecnología (Fondecyt) y, por tanto, sus proyectos deben presentarse al alero de otros grupos de estudio. Esta situación es anacrónica con relación al desarrollo de investigación y su ubicación en países como Estados Unidos, Canadá o Brasil.

\section{La organización universitaria y la formación de posgrado}

La diferencia directriz temático-profesional/disciplinar tiene también consecuencias para los procesos pedagógicos que acontecen en cada espacio. En los posgrados de orientación principalmente disciplinar, la motivación central no viene de la institución universitaria que cobija al programa; no se trata de instrumentos de negocio ni constitución de identidades organizacionales. Más bien se trata de espacios de investigación y discusión, en los cuales tanto académicos como estudiantes realizan sus propias vocaciones académicas y teóricas. Se trata de ámbitos en los cuales, mediante el desarrollo personal, se contribuye a la disciplina. En los posgrados de orientación predominantemente temático-profesional el interés se centra en el cliente y en los productos que ese espacio pueda ofrecerle, asociados sobre todo a las herramientas disociadas de su dimensión teóricosustantiva. 
Para el proceso pedagógico esto implica dos modos radicalmente distintos de constituirse la relación enseñanza-aprendizaje. En los primeros importa menos el currículum, las herramientas, y más el proceso de discusión que se forma en una comunidad académica; en los segundos hay una tendencia predominante a recargar con cursos y con materiales. Allí la formación de posgrado temático no es muy distinta a la de pregrado, en el sentido de que existe un profesor que transmite su conocimiento y un grupo de estudiantes que lo recibe de modo pasivo. Esto implica que no hay un cambio de lógica; no se observa el posgrado como un lugar donde ocurren cosas distintas en términos pedagógicos.

\section{Posgrados en ciencias sociales en Chile: propuestas de regulación}

A partir de este diagnóstico que permite la distinción temáticoprofesional/disciplinar, es posible establecer recomendaciones dirigidas a la formación de mecanismos regulatorios para monitorear y mejorar la calidad de la oferta de posgrado en ciencias sociales en Chile.

\section{Claustro académico}

Una instancia que contribuye decisivamente a la calidad de los posgrados en ciencias sociales es la descentralización de su dirección académica y administrativa mediante la formación de un claustro, orientado a la supervisión de las decisiones estratégicas del programa, por ejemplo:

- Número de vacantes.

- Requisitos de admisión adecuados a los objetivos del programa.

- Orientaciones generales para la publicidad del programa.

- Decisiones de aceptación en casos dudosos.

- Decisiones referidas a la promoción académica de los estudiantes.

Este claustro, cuando existe, está formado por académicos de alto reconocimiento en el campo disciplinar de que se trate. 


\section{Incentivar el desarrollo de programas de posgrado disciplinares}

Probablemente, una de las más importantes recomendaciones regulatorias es la que se relaciona con el desarrollo de mecanismos de incentivo para la formación de programas de posgrado de carácter disciplinar. Como se ha mencionado, existe una estructura política y económica que ya privilegia el desarrollo de posgrados temáticos. Por otra parte, la historia chilena de los últimos cuarenta años es altamente decisiva en la limitación del desarrollo disciplinar en el área de las ciencias sociales. Por ello, es preciso establecer mecanismos de incentivo al desarrollo de programas que contribuyan al despliegue de disciplinas sociales fuertes en el ámbito de los conocimientos teóricos, la investigación y el intercambio disciplinar internacional. Ellos podrán contribuir a la consolidación de las ciencias sociales en Chile porque permiten:

- Formar investigadores con conocimientos disciplinares sólidos.

- Generar identidades disciplinares acopladas con los modos internacionales de hacer disciplina.

- Un mejor acercamiento a las posibilidades de interdisciplinariedad, en tanto se disponga de investigadores formados sólidamente en sus disciplinas, en lo que comparten y en lo que las diferencia de otras.

- Un mejor conocimiento de los problemas temáticos en el país y en la región. En este sentido, el incentivo contribuye también decisivamente a mejores programas temáticos, los que podrán ser abordados con un conocimiento disciplinar más sólido.

- Distinguir entre intereses políticos e intereses académicos en la formación de posgrado, lo que puede incluso comenzar a superar la distinción temático-profesional/disciplinar.

\section{Financiamiento de posgrado}

En conexión con lo anterior, es necesaria una política de financiamiento para el desarrollo de los posgrados que quiebre las barreras corporativas establecidas del nivel nacional e incentive a los existentes o a los nuevos posgrados su vinculación con el medio internacional. 
El financiamiento de posgrado debe permitir que aquellos que estudien en el país accedan a otros entornos culturales y se conecten con discusiones, formas de trabajo y conocimientos que se desarrollan en otros lugares del mundo. Ciertamente, es responsabilidad de los posgrados en ciencias sociales en Chile lograr una alta calidad académica que incentive a los estudiantes chilenos y extranjeros a formarse en el país, pero ello puede lograrse también con una política de financiamiento que incentive, por ejemplo:

- Intercambio académico de los investigadores con centros internacionales en la disciplina o en el ámbito temático de que se trate.

- Intercambio de estudiantes con centros internacionales en los cuales problemáticas disciplinares o temáticas similares se estén investigando.

- Mayor cantidad de becas para estudiantes nacionales que opten por estudiar en el país en programas de posgrado de calidad reconocida y comprobada.

- Financiamiento de bases de datos disciplinares y para la compra de material bibliográfico nacional e internacional.

- Financiamiento de las adecuaciones en conectividad de las instituciones universitarias donde se imparten posgrados de calidad, de manera de incentivar prácticamente el desarrollo de redes de investigadores y estudiantes en las disciplinas o ámbitos temáticos respectivos.

- Pasantías para profesores y estudiantes en diversas partes del mundo en las cuales las materias de investigación relevante lo justifiquen.

- Mayores posibilidades de financiamiento para el desarrollo de encuentros internacionales en Chile.

- Convenios generales con universidades extranjeras.

\section{Fortalecimiento de unidades académicas}

Importante también, en un sentido de política de financiamiento universitario con especial repercusión en el área de posgrado, es el fortalecimiento de las unidades académicas que están tras 
170 LOS DESAFÍOS DE LOS POSGRADOS EN CIENCIAS SOCIALES EN CHILE Teresa Matus, Aldo Mascareño, Adriana Kaulino

estos programas. Se requiere un equipo académico idóneo para su desarrollo. Esto implica, por ejemplo, el financiamiento de costos de reemplazo para profesores, de manera que académicos nacionales puedan dedicarse por un periodo extenso a la investigación y su puesto en el posgrado sea cubierto con alguien de la misma calidad. Esto repercute en la calidad y novedad de la docencia, en la actualización de los investigadores, en la productividad académica y en una mejor formación de los estudiantes de posgrado, sea en programas temáticos o disciplinares.

Por otro lado, se requiere también que las universidades logren definir claramente cuál es su política de posgrado: si se trata de programas temáticos o disciplinares o de ambos. Para incentivar esta autodefinición, los marcos regulatorios debieran, entre otros aspectos:

- Restringir la tendencia a la creación de posgrados sólo por un interés institucional de financiamiento.

- Limitar la creación de posgrados en espacios académicos que no cuentan con comunidades académicas suficientes para el logro de los objetivos que el programa se propone.

- Incentivar una coherencia clara entre las comunidades académicas que son la base de un programa y las características y sentido de ese programa de posgrado.

\section{La complejidad de la estructura de posgrados en ciencias sociales en Chile}

Una afirmación paradójica puede ser útil para iniciar la formulación de esta síntesis: el desarrollo de modelos complejos para la evaluación de la calidad de programas de posgrado en ciencias sociales en Chile requiere mirar más allá del país. Como otros tipos de espacios sociales y educativos en el contexto de la sociedad nacional, los estudios y programas de posgrado en Chile se ven tensionados por una referencia hacia intereses temáticos y también disciplinares. En tanto las disciplinas de las ciencias sociales (psicología, sociología, ciencia política, antropología, trabajo social) pertenecen al horizonte 
general de las disciplinas científicas, su modo de funcionamiento transciende las fronteras regionales y nacionales, y aspiran de ese modo a la aplicabilidad universal de sus métodos y teorías.

Los posgrados en ciencias sociales en Chile oscilan entre esas dos modalidades de observación: el énfasis temático-profesional y el énfasis disciplinar. Una consecuencia inmediata de este planteamiento es asociar los programas de posgrado de tipo temático predominantemente a la satisfacción de intereses no estrictamente académicos, sino políticos o profesionales. En forma paralela, esto supone vincular los programas de posgrado de tipo disciplinar a los problemas científicos que las mismas disciplinas crean en sus propios procesos de investigación.

Este tensionamiento de la estructura de posgrados en ciencias sociales hacia lo temático es problemática por dos razones centrales. La primera es que, con una demanda exclusiva de este tipo, las organizaciones universitarias comienzan a entender los estudios de posgrado en ciencias sociales como una forma de financiamiento. Éstos se transforman en un negocio que subvalora su dimensión de investigación académica y de producción de conocimientos que contribuyan al desarrollo de la disciplina. En tanto el programa de posgrado busque entregar un producto a alguien que espera recibirlo, el proceso pedagógico se convierte en una transmisión unilateral de herramientas y conocimientos que no promueven la formación y producción individual de estos mismos.

La segunda razón por la cual el tensionamiento de la estructura de posgrados en ciencias sociales hacia lo temático es problemática, se relaciona con la pretensión académica de esta clase de posgrados en Chile. Para afirmarla, proponen la entrega de herramientas metodológicas para el abordaje de distintos temas. Esos métodos son, por cierto, un resultado evolutivo de los procesos de investigación y desarrollo de las distintas disciplinas que los han creado y, en ese sentido, son indisociables de las teorías que se despliegan en ellas. Lo que realiza la pretensión académica de los posgrados temáticos 
es adoptar esas metodologías de la dimensión disciplinar, pero sin referencia a la teoría sustantiva que las apoya. Los métodos se convierten en una técnica al servicio de objetivos no sustentados teóricamente y que, tratándose de posgrados temáticos, vienen definidos por intereses políticos o económicos.

Frente a esta situación, es importante la modificación de marcos regulatorios, con el fin de reconsiderar los programas de posgrado en ciencias sociales en Chile como una instancia de formación de investigadores que generen nuevos conocimientos, en contacto con el horizonte mundial de las disciplinas y las problemáticas sociales de interés, ya sea científico, político o profesional. Todo ello para que, paralelamente, en los casos en que ese sea el objetivo, realizar intervenciones sociales no sólo a partir de técnicas, sino también de los criterios sustantivos que puede entregar el desarrollo teórico de las diversas disciplinas en campos temáticos diferenciados. Sólo de ese modo los programas de posgrado en ciencias sociales en Chile podrán trascender las determinaciones e intereses locales y abordar esos mismos intereses con la perspectiva de la sociedad mundial en la cual están insertos.

\section{Referencias bibliográficas}

Aedo, C. y González, L. (2004) La educación superior en Chile. Calidad en la Educación, 21, pp. 61-85.

Bernasconi, A. y Rojas, F. (2004) Informe sobre la educación superior en Chile. Versión digital.

Chernilo, D. (2007) A Social Theory of the Nation State. London: Routledge.

Chernilo, D. y Mascareño, A. (2005) Universalismo, particularismo y sociedad mundial: Obstáculos y perspectivas de la sociología latinoamericana. Persona y Sociedad, XIX(3), pp. 17-45.

Habermas, J. y Rawls, J. (1998) Debate sobre el liberalismo politico. Madrid: Alianza.

Habermas, J. (2000) Facticidad y validez. Madrid: Trotta. 
Kaulino, A. (2001) La invisibilidad del saber psicológico. Revista Perspectivas, U. Cardenal Raúl Silva Henríquez, Santiago de Chile.

Kaulino, A. La vocación social de la psicología. Revista Retrospectivas en Psicología, Santiago de Chile.

Larrain, J. (1989) Theories of development. Capitalism, colonialism and dependency. Cambridge: Polity Press.

Lemaitre, M. (2004) Mecanismos de aseguramiento de la calidad: respuesta a los desafíos del cambio en la educación superior. Calidad en la Educación, 21, pp. 87-106.

Luhmann, N. \& Schorr E. (1979) Reflexionsprobleme im Erziehungssystem. Stuttgart: Klett-Cotta.

Luhmann, N. y Di Giorgi, R. (1998) Teoría de la sociedad. México D.F: Universidad Iberoamericana, Triana.

Luhmann, N. (1971) Sinn als Grundbegriff der Soziologie. En Habermas J. \& Luhmann, N. Theorie der Gesellschaft oder Sozialtechnologie. Frankfurt: Suhrkamp, pp. 25-100.

Mascareño, A. (2006) Sociología de la cultura. Estudios Públicos, 105, pp. 61-112.

Mascareño, A. (2007) Sociología de la intervención. En: Mediación y complejidad. Santiago de Chile: Universidad Diego Portales.

Matus, T. (2003) La intervención social bajo el resplandor de lo público. En: Hoy es mi tiempo. Santiago de Chile: FOSIS, Gobierno de Chile.

Matus, T. (2003) La intervención social como gramática. Revista Trabajo Social, 71 .

Mereminskaya, E. y Mascareño, A. (2005) La desnacionalización del derecho y la formación de regímenes globales de gobierno. En Martinic, M.D. (ed.) Sesquicentenario del Código Civil de Andrés Bello: Pasado, presente y futuro de la codificación. Santiago de Chile: Lexis-Nexis, pp. 13911427.

Rawls, J. (2002) La justicia como equidad. Una reformulación. Barcelona: Paidós.

Rodríguez, D. (2004) Organizaciones para la modernización. México D.F.: Universidad Iberoamericana.

Stichweh, R. (2000) Die Weltgesellschaft. Soziologische Analysen. Frankfurt: Suhrkamp. 
174 LOS DESAFÍOS DE LOS POSGRADOS EN CIENCIAS SOCIALES EN CHILE -

Teresa Matus, Aldo Mascareño, Adriana Kaulino

Veugelers, W. y Zijlstra, H. (2002) Redes de escuelas: una herramienta poderosa. Cuadernos de Pedagogía, 319, pp. 64-68.

Willke, H. (1993) Systemtheorie entwickelter Gesellschaften. Dynamik und Riskanz moderner gesellschaftlicher Selbstorganisation. Weinheim, München: Juventa.

Willke, H. (1984) Soziale Systeme. Frankfurt: Suhrkamp.

Willke, H. (1996a) Ironie des Staates. Frankfurt: Suhrkamp.

Willke, H. (1996b) Systemtheorie II: Interventionstheorie. Stuttgart: Lucius \& Lucius Verlag.

Willke, H. (1997ª) Die Gesellschaft der Gesellschaft. Frankfurt: Suhrkamp.

Recibido: 23 de enero de 2008

Aprobado: 9 de abril de 2008 